\title{
COMPACT AND WEAKLY COMPACT HOMOMORPHISMS BETWEEN ALGEBRAS OF DIFFERENTIABLE FUNCTIONS
}

\author{
MANUEL GONZÁLEZ AND JOAQUÍN M. GUTIÉRREZ
}

(Communicated by Palle E. T. Jorgensen)

\begin{abstract}
It is proved that the weakly compact continuous homomorphisms between algebras of $k$-times continuously differentiable functions on Banach spaces are induced by constant mappings.
\end{abstract}

Recently many authors have studied compact and weakly compact homomorphisms between function algebras. Lindström and Llavona [5] treat weakly compact continuous homomorphisms tetween algebras of the type $C(T)$, where $T$ is a completely regular Hausdorff space; Feldman [1] deals with compact composition operators on some function spaces, and Kamowitz [4] describes the compact endomorphisms of $C^{1}[0,1]$.

Llavona asked whether the results in [5] are valid in the case of algebras of differentiable functions on Banach spaces. The purpose of this note is to give an affirmative answer to this question by proving that weakly compact continuous homomorphisms between algebras of differentiable functions are induced by constant mappings. The difficulty we face is that in [5] the existence of continuous functions separating points and closed sets plays an essential role, while in the differentiable case these functions do not exist in general. In this note we deal with Fréchet differentiability, but our results are also valid for Hadamard differentiable functions.

$\mathbb{R}$ denotes the real field and $\mathbb{N}$ the nonnegative integers. $E$ and $F$ are real Banach spaces, $E^{*}$ the topological dual of $E$, and $C^{k}(E)$ the space of all real-valued $k$-times continuously Fréchet differentiable functions on $E$. Two topologies are natural on $C^{k}(E)$ (see e.g. [6]):

(a) The compact open topology of order $k$ given by the seminorms:

$$
p_{L}(f)=\sup \left\{\left\|d^{j} f(x)\right\|: x \in L ; 0 \leq j \leq k\right\},
$$

where $d^{0} f=f, d^{j} f$ is the $j$ th derivative of $f$, and $L$ runs through the compact subsets of $E$. We recall that $d^{j} f$ is a continuous mapping from $E$

Received by the editors January 21, 1991.

1991 Mathematics Subject Classification. Primary 46E25, 26E15.

Key words and phrases. Composition operators, (weakly) compact homomorphisms, algebras of differentiable functions.

The first author was supported in part by DGICYT Grant PB88-0417.

The second author was supported in part by DGICYT Grant PB87-1031. 
into the space $\mathscr{P}\left({ }^{j} E\right)$ of real-valued $j$-homogeneous continuous polynomials on $E$.

(b) The compact-compact topology of order $k$, given by the seminorms:

$$
q_{L}(f)=\sup \left\{|f(x)|,\left|d^{j} f(x)(y)\right|: x, y \in L ; 1 \leq j \leq k\right\},
$$

where $L$ is allowed to range on the compact subsets of $E$.

Both topologies coincide only when $E$ is finite dimensional.

When we say that an algebra homomorphism $A: C^{k}(E) \rightarrow C^{k}(F)$ is continuous, we understand that both $C^{k}(E)$ and $C^{k}(F)$ are endowed with one of the natural topologies. In [3] it is proved that any nonzero continuous homomorphisms $A$ as above is of the form $A f:=f \circ \varphi\left(f \in C^{k}(E)\right)$, where $\varphi: F \rightarrow E$ satisfies $\phi \circ \varphi \in C^{k}(F)$ for each $\phi \in E^{*}$ (and so, if $\operatorname{dim}(E)<\infty$, then $\left.\varphi \in C^{k}(F)\right)$. For completeness, we sketch the proof. First, by standard arguments (see e.g. [2]), it can be shown that, for any nonzero continuous homomorphism $\Phi: C^{k}(E) \rightarrow \mathbb{R}$, there is a unique $x \in E$ such that $\Phi(f)=f(x)$ for every $f \in C^{k}(E)$. Now, let $\delta_{y}: C^{k}(F) \rightarrow \mathbb{R}$ be the evaluation map at $y \in F$. Then $\delta_{y} \circ A$ is a nonzero continuous homomorphism from $C^{k}(E)$ to $\mathbb{R}$, so there is a unique $x \in E$ such that $A f(y)=\delta_{y} \circ A f=f(x)$ for every $f \in C^{k}(E)$. To finish the proof, it is enough to let $\varphi: F \rightarrow E$ be the map taking $y$ to $x$. For a wide class of Banach spaces $E$ (including all the separable spaces and their duals), any algebra homomorphism $A: C^{k}(E) \rightarrow C^{k}(F)$ is automatically continuous [3].

Following [5] we say that a homomorphism $A: C^{k}(E) \rightarrow C^{k}(F)$ is (weakly) compact if it maps bounded subsets of $C^{k}(E)$ into relatively (weakly) compact subsets of $C^{k}(F)$.

We first prove the result in the case $E=F=\mathbb{R}$, and then derive the general case from it.

1. Proposition. Let $A: C^{k}(\mathbb{R}) \rightarrow C^{k}(\mathbb{R})$ be a nonzero algebra homomorphism, for some $k \in \mathbb{N} \backslash\{0\}$, and let $\varphi \in C^{k}(\mathbb{R})$ be its inducing function. Then the following assertions are equivalent:

(a) $\varphi$ is constant;

(b) A has one-dimensional rank;

(c) $A$ is compact;

(d) $A$ is weakly compact.

Proof. (a) $\Rightarrow$ (b). If $\varphi(y)=x_{0}$ for every $y \in \mathbb{R}$, then $A f=f\left(x_{0}\right) \cdot \mathbf{1}$ $\left(f \in C^{k}(\mathbb{R})\right)$, where 1 is the constant function with value 1 .

(b) $\Rightarrow($ c) $\Rightarrow($ d) . They are obvious.

(d) $\Rightarrow$ (a). If $\varphi$ is not constant, then we may assume there exist $a<b$ such that $\varphi(a)<\varphi(b), \varphi$ is increasing in $[a, b]$ and $\varphi^{\prime}(x) \geq m>0$ for each $x \in[a, b]$.

Let $\psi:=\left.\varphi\right|_{[a, b]}$, and let $B: C^{k}(\mathbb{R}) \rightarrow C^{k}[a, b]$ denote the homomorphism given by $B f=f \circ \psi \quad\left(f \in C^{k}(\mathbb{R})\right)$.

Choose a sequence $\left(x_{n}\right)$ with:

$$
\psi(a)<x_{1}<\cdots<x_{n}<\cdots<\psi(b),
$$

and $\left|x_{n+1}-x_{n}\right| \leq h<1$ for every $n \in \mathbb{N}$. 
For each $n \in \mathbb{N}$, we can find a function $f_{n} \in C^{k}(\mathbb{R})$ supported by $\left(x_{n}, x_{n+1}\right)$, with $\left\|f_{n}^{(k)}\right\|_{\infty}=1$. Therefore, we have $\left\|f_{n}^{(k-j)}\right\|_{\infty}<h^{j} \quad(1 \leq j \leq k)$.

If $y_{i}=\psi^{-1}\left(x_{i}\right)$, for every $i \in \mathbb{N}$, we then have:

$$
a<y_{1}<\cdots<y_{n}<\cdots<b,
$$

and $f_{n} \circ \psi$ is supported by $\left(y_{n}, y_{n+1}\right) \quad(n \in \mathbb{N})$.

The sequence $\left(f_{n}\right)$ is equivalent to the unit vector basis of $c_{0}$. Indeed, if $\left(a_{n}\right)$ is a finite sequence of real numbers and $\|\cdot\|$ denotes the norm in the Banach space $C^{k}[\psi(a), \psi(b)]$, then since $\left(f_{n}\right)$ have disjoint supports, we have:

$$
\left\|\sum a_{n} f_{n}\right\|=\sup \left\{\left|\sum a_{n} f_{n}^{(j)}(t)\right|: 0 \leq j \leq k ; t \in[\psi(a), \psi(b)]\right\}=\sup _{n}\left|a_{n}\right| .
$$

The sequence $\left(B f_{n}\right)$ is also equivalent to the basis of $c_{0}$. Again, for every finite sequence $\left(a_{n}\right)$ of real numbers, since $\left(f_{n} \circ \psi\right)$ have disjoint supports, we have:

$$
\begin{aligned}
\left\|\sum a_{n} B f_{n}\right\| & =\left\|\sum a_{n} f_{n} \circ \psi\right\| \\
& =\sup \left\{\left|\sum a_{n}\left(f_{n} \circ \psi\right)^{(j)}(t)\right|: 0 \leq j \leq k ; a \leq t \leq b\right\} \\
& =\sup _{n} \sup \left\{\left|a_{n}\right| \cdot\left|\left(f_{n} \circ \psi\right)^{(j)}(t)\right|: 0 \leq j \leq k ; y_{n}<t \leq y_{n+1}\right\} \\
& =\sup _{n}\left|a_{n}\right| \cdot \sup \left\{\left|\left(f_{n} \circ \psi\right)^{(j)}(t)\right|: 0 \leq j \leq k ; a \leq t \leq b\right\} \\
& \leq M \cdot \sup _{n}\left|a_{n}\right|
\end{aligned}
$$

for some $M>0$. Moreover,

$$
\begin{aligned}
\sup \left\{\left|\left(f_{n} \circ \psi\right)^{(j)}(t)\right|: 0 \leq j \leq k ; a \leq t \leq b\right\} & \\
\geq \sup \left\{\left|f_{n}^{(k)}(\psi(t))\right| \cdot\left|\psi^{\prime}(t)\right|^{k}\right. & \\
& \left.\quad-\left|\sum_{j=1}^{k-1} b_{j} f_{n}^{(j)}(\psi(t)) \psi^{\left(i_{1}\right)}(t) \cdots \cdots \psi^{\left(i_{j}\right)}(t)\right|: a \leq t \leq b\right\},
\end{aligned}
$$

where $\left(b_{j}\right)$ are the constants given by the chain rule of order $k$ (see for instance [7, I.7.10 Corollary]) and $i_{1}, \ldots, i_{j}$ are positive integers with $i_{1}+\cdots+i_{j}=k$.

We can modify $h$ if necessary, so that:

$$
\left|\sum_{j=1}^{k-1} b_{j} h^{j} \psi^{\left(i_{1}\right)}(t) \cdots \cdots \psi^{\left(i_{j}\right)}(t)\right| \leq \frac{m^{k}}{2} \quad(t \in[a, b]) .
$$

Then

$$
\sup \left\{\left|\left(f_{n} \circ \psi\right)^{(j)}(t)\right|: 0 \leq j \leq k ; a \leq t \leq b\right\} \geq m^{k} / 2
$$

and

$$
\left\|\sum a_{n} B f_{n}\right\| \geq \frac{m^{k}}{2} \cdot \sup _{n}\left|a_{n}\right| .
$$

Therefore, $B$ preserves a copy of $c_{0}$, and hence, it is not weakly compact. Let now $R: C^{k}(\mathbb{R}) \rightarrow C^{k}[a, b]$ be the restriction homomorphism. Then we have $R \circ A=B$, so $A$ is not weakly compact. 
2. Theorem. Given $k \in \mathbb{N} \backslash\{0\}$, let $A: C^{k}(E) \rightarrow C^{k}(F)$ be a nonzero continuous algebra homomorphism when $C^{k}(E), C^{k}(F)$ are both endowed with one of the natural topologies, and let $\varphi: F \rightarrow E$ be its inducing function. Then the following assertions are equivalent:

(a) $\varphi$ is constant;

(b) $A$ has one-dimensional rank;

(c) $A$ is compact;

(d) $A$ is weakly compact.

Proof. (a) $\Rightarrow$ (b) like in Proposition 1.

(b) $\Rightarrow(\mathrm{c}) \Rightarrow(\mathrm{d})$. They are clear.

(d) $\Rightarrow$ (a). Suppose $\varphi$ is not constant. Then there is $y_{0} \in F$ with $\varphi\left(y_{0}\right) \neq$ $\varphi(0)$. Choose $x^{*} \in E^{*}$ such that $\left\langle\varphi(0), x^{*}\right\rangle \neq\left\langle\varphi\left(y_{0}\right), x^{*}\right\rangle$, and let $i: \mathbb{R} \rightarrow F$ be given by $i(\lambda)=\lambda y_{0}$. If $\bar{\varphi}=x^{*} \circ \varphi \circ i$, then $\bar{\varphi}$ belongs to $C^{k}(\mathbb{R})$ and satisfies $\bar{\varphi}(0) \neq \bar{\varphi}(1)$. Let $\bar{A}: C^{k}(\mathbb{R}) \rightarrow C^{k}(\mathbb{R})$ be the homomorphism given by $\bar{A} f=f \circ \bar{\varphi}$. By the previous proposition, $\bar{A}$ is not weakly compact. If $I: C^{k}(\mathbb{R}) \rightarrow C^{k}(E)$ is defined by $I g=g \circ x^{*}\left(g \in C^{k}(\mathbb{R})\right)$ and $R: C^{k}(F) \rightarrow$ $C^{k}(\mathbb{R})$ is the restriction homomorphism given by $R f=f \circ i$, then $\bar{A}=R \circ A \circ I$. Since $\bar{A}$ is not weakly compact, neither is $A$.

3. Remark. For $k \in \mathbb{N}$, let $C_{w u}^{k}(E)$ denote the class of all $f \in C^{k}(E)$ such that $f, d^{j} f$, and $d^{j} f(x) \quad(x \in E, 1 \leq j \leq k)$ are weakly uniformly continuous on bounded subsets of $E$. In $[6,11.2 .6]$ it is proved that if $E^{*}$ has the bounded approximation property, then every nonzero algebra homomorphism $A: C_{w u}^{k}(E) \rightarrow C_{w u}^{k}(F)$ is automatically continuous and induced by a function $\varphi: F \rightarrow E^{* *}$ such that $\phi \circ \varphi \in C_{w u}^{k}(F)$ for each $\phi \in E^{*}$. Applying the proof of Theorem 2 to this case, we conclude that $A$ is weakly compact if and only if $\varphi$ is constant. For $k=0$, this result is contained in [5].

4. Remark. Theorem 2 fails for homomorphisms $A: C^{k}(E) \rightarrow C^{m}(F)$ with $k>m$. Indeed, suppose $\varphi: F \rightarrow E$ is a nonconstant mapping that factors through a finite-dimensional Banach space $G$ :

$$
F \stackrel{\xi}{\rightarrow} G \stackrel{\zeta}{\rightarrow} E \quad(\varphi=\zeta \circ \xi),
$$

where $\xi$ is of class $C^{m}$ and $\zeta$ is of class $C^{k}$ ( $k$ finite or infinite, $k>m$ ). Then the homomorphism $A: C^{k}(E) \rightarrow C^{m}(F)$ given by $A f=f \circ \varphi(f \in$ $\left.C^{k}(E)\right)$ factors in the following way:

$$
C^{k}(E) \stackrel{A_{\xi}}{\rightarrow} C^{k}(G) \stackrel{i}{\rightarrow} C^{m}(G) \stackrel{A_{\xi}}{\rightarrow} C^{m}(F),
$$

where

$$
\begin{aligned}
& A_{\zeta}(f)=f \circ \zeta \quad\left(f \in C^{k}(E)\right), \\
& A_{\xi}(g)=g \circ \xi \quad\left(g \in C^{m}(G)\right),
\end{aligned}
$$

and $i$ is the identity map, which is known to be compact. So, $A$ is compact too.

An analogous remark can be made for $k=m=\infty$.

However, for $k<m$ every continuous homomorphism from $C^{k}(E)$ to $C^{m}(F)$ is induced by a constant mapping $F \rightarrow E$ (see $[6,11.2 .7]$ ). 


\section{REFERENCES}

1. W. Feldman, Compact weighted composition operators on Banach lattices, Proc. Amer. Math. Soc. 108 (1990), 95-99.

2. J. Gómez, Espectro e ideales primarios del álgebra $C_{w b}^{p}(E)$ de funciones débilmente diferenciables sobre un espacio de Banach, Rev. Real Acad. Cienc. Exact. Fís. Natur. Madrid 75 (1981), 514-519.

3. J. M. Gutiérrez and J. G. Llavona, Composition operators between algebras of differentiable functions, Trans. Amer. Math. Soc. (to appear).

4. H. Kamowitz, Compact endomorphisms of Banach algebras, Pacific J. Math. 89 (1980), 313-325.

5. M. Lindström and J. G. Llavona, Compact and weakly compact homomorphisms between algebras of continuous functions, J. Math. Anal. Appl. (to appear).

6. J. G. Llavona, Approximation of continuously differentiable functions, Math. Studies, no. 130, North-Holland, Amsterdam, 1986.

7. P. ver Eecke, Fondements du calcul différentiel, PUF, Paris, 1983.

Departamento de Matemáticas, Facultad de Ciencias, Universidad de Cantabria, 39071 Santander, SPain

Departamento de Matemática Aplicada, ETS de Ingenieros Industriales, Universidad Politécnica de Madrid, C. José Gutiérrez Abascal 2, 28006 Madrid, Spain 